\title{
Interleukin-6 and rs1800796 locus single nucleotide polymorphisms in response to hypoxia/reoxygenation in hepatocytes
}

\author{
ZHAOWEN WANG ${ }^{*}$, SHAOHAN WU* , JIANHUA LIAO, LIN ZHONG, \\ TONGHAI XING, JUNWEI FAN and ZHIHAI PENG \\ Department of General Surgery, Shanghai General Hospital, \\ Shanghai Jiao Tong University School of Medicine, Shanghai 200080, P.R. China
}

Received November 5, 2015; Accepted May 10, 2016

DOI: $10.3892 / \mathrm{ijmm} .2016 .2595$

\begin{abstract}
Ischemia-reperfusion injury due to hypoxia/reoxygenation $(H / R)$ is one of the main causes of liver damage during liver surgery. Donor interleukin-6 (IL-6) rs1800796 single nucleotide polymorphisms (SNPs) affect the metabolism of tacrolimus following liver transplantation-related hepatic H/R. This study investigated the response of $I L-6$ and its promoter polymorphisms to hepatic H/R in liver parenchymal cells. The association between $I L-6$ rs1800796 SNPs and IL-6 expression was measured in 84 disease-free liver tissues using tissue microarrays and immunohistochemistry. Subsequently, LO2G, LO2C and NC-LO2 cells were successfully constructed via stable lentivirus-mediated transfection. The effects of $I L-6$ and its SNPs on the biological function of LO2 cells were examined using a cell model of H/R. Our results revealed that IL-6 was mainly expressed in hepatocytes. The intermediate IL-6 expression rate in genotype $\mathrm{CC}$ carriers was higher than that in genotype $\mathrm{CG} / \mathrm{GG}$ carriers $(\mathrm{P}=0.006)$, which was subsequently verified at the $I L-6$ mRNA level $(\mathrm{P}=0.002)$. The concentrations of alanine aminotransferase in the $\mathrm{LO} 2 \mathrm{G}$ cells were significantly higher than those in the LO2C cells following H/R for $6 \mathrm{~h}$ and $\mathrm{H} / \mathrm{R}$ for $24 \mathrm{~h}(\mathrm{P}<0.05)$. The viability of the $\mathrm{LO} 2 \mathrm{C}$ cells was higher than that of the LO2G cells $(\mathrm{P}<0.05)$. Furthermore, the expression of $I L-6$ and its downstream molecules was significantly increased in the LO2C cells compared with the LO2G cells $(\mathrm{P}<0.05)$. Therefore, the sequence variants of rs1800796 SNPs $(\mathrm{G} \rightarrow \mathrm{C})$ exhibit an increased $I L-6$ transcription efficiency in liver parenchymal cells. In addition, the increased expression of $I L-6$ protects the hepatocytes following hepatic H/R injury.
\end{abstract}

Correspondence to: Dr Junwei Fan or Dr Zhihai Peng, Department of General Surgery, Shanghai General Hospital, Shanghai Jiao Tong University School of Medicine, 100 Haining Road, Hongkou, Shanghai 200080, P.R. China

E-mail: fjwnet@163.com

E-mail:wshwshnet@163.com

*Contributed equally

Key words: hypoxia/reoxygenation, interleukin-6, single nucleotide polymorphism

\section{Introduction}

Ischemia-reperfusion injury (IRI) caused by hypoxia/reoxygenation $(\mathrm{H} / \mathrm{R})$ in the liver is a common cause of liver damage during liver resection, liver transplantation and hepatic failure (1-4). It has been widely demonstrated that interleukin-6 (IL-6) is a vital mediator, and IL-6/signal transducer and activator of transcription 3 (STAT3) signaling is of major importance in restoring normal hepatic function following liver IRI (5-7). Studies have found that IL- 6 is transported to the liver in an endocrine manner from immunocytes at the wound site, or in a paracrine manner from hepatic immunocytes within the liver $(8,9)$. However, the biological mechanisms of $I L-6$ synthesis in parenchymal hepatic cells, not hepatic immunocytes, has previously been overlooked.

Additionally, the prevalence of rs1800795 (-174G/C) and rs1800797 locus polymorphisms in the $I L-6$ gene promoter is extremely rare, whereas the allele $C$ at the $I L-6$ rs 1800796 locus is frequently observed in East Asian populations (10-13). Although studies have reported that $I L-6$ rs1800796 (also designated as $-572 \mathrm{C} / \mathrm{G}$ or $-634 \mathrm{G}>\mathrm{C}$ ) single nucleotide polymorphisms (SNPs) affect $I L-6$ transcriptional activity, their conclusions were not always consistent among similar populations $(13,14)$. The rs1800796 locus SNPs may be present in a regulatory DNA binding site, leading to altered affinity with the regulatory protein, and may subsequently influence $I L-6$ transcription efficiency (15). Furthermore, only donor $I L-6$ rs1800796 SNPs affect the metabolism of tacrolimus, an immunosuppressive drug, mainly by altering liver function and regeneration in response to hepatic H/R following liver transplantation (16). Therefore, we hypothesized that $I L-6$ and its rs1800796 SNPs in parenchymal hepatic cells may protect liver function during $\mathrm{H} / \mathrm{R}$, and that the sequence variants of rs1800796 SNPs $(\mathrm{G} \rightarrow \mathrm{C})$ may increase the transcription efficiency of $I L-6$. Thus, in order to confirm our hypothesis, in the present study, we investigated the response of $I L-6$ and its rs1800796 locus SNPs to hepatic $\mathrm{H} / \mathrm{R}$ stimulus in parenchymal liver cells.

\section{Materials and methods}

Collection of liver tissue samples. Disease-free liver tissues were collected from 84 male Chinese volunteers at the 
Shanghai Jiao Tong University Affiliated First People's Hospital (Shanghai, China) after obtaining written informed consent. The mean age of the subjects was $35.8 \pm 8.3$ years. The subjects had no hepatic disease, cardiovascular or cerebrovascular disease that affected the serum IL-6 levels. Each sample was immediately cryopreserved in liquid nitrogen, and then transferred to refrigerator at $-80^{\circ} \mathrm{C}$. All the surgical protocols were conducted similarly. This study was conducted according to the Declaration of Helsinki and its amendments, and was approved by the Ethics Committee of the Medical Faculties of Shanghai Jiao Tong University.

Materials. Rosewell Park Memorial Institute (RPMI)-1640 medium and fetal bovine serum (FBS) were purchased from Gibco (Grand Island, NY, USA). Dulbecco's modified Eagle's medium (DMEM), TRIzol reagemt and the SuperScript VILO cDNA Synthesis kit were purchased from Invitrogen (Carlsbad, CA, USA). Wild-type overexpressed $I L-6$ vectors named p-IL-6(WT), negative control $I L-6$ overexpression vectors, HG transgene reagent, all lentivectors, and the Lentiviral Packaging Plasmid Mix (Lenti-HG Mix) were obtained from GenomeDitech Co., Ltd. (Shanghai, China). Restriction endonucleases (BamHI, ClaI and EcoRI) were purchased from MBI Fermentas (MBI Fermentas, Burlington, ON Canada). The QuickChange $^{\mathrm{TM}}$ Site-Directed Mutagenesis kit was obtained from Stratagene (La Jolla, CA, USA). The RNeasy mini kits and the AllPrep DNA/RNA Mini kits were obtained from Qiagen (Venlos, The Netherlands). The SuperScript VILO cDNA synthesis kits were obtained from Invitrogen. The cell counting kit-8 (CCK-8) and alanine aminotransferase (AST) enzymelinked immunosorbent assay (ELISA) kits were purchased from R\&D Systems (Minneapolis, MN, USA). The antibodies used were as follows: anti-IL- 6 and anti-STAT3 polyclonal antibodies (ab6672 and ab68153; Abcam, Cambridge, UK). Ampicillin, puromycin, or blasticidin were obtained from MP Biomedicals (Irvine, CA, USA). The secondary antibody [goat anti-rabbit immunoglobulin G (ZB-2301)] was obtained from Golden Bridge Biotechnology Co., Ltd. (Beijing, China). The human normal liver cell line LO2 (17) and 293T cells were purchased from the Shanghai Institute of Cell Biology (Shanghai, China) and the cells were cultured in DMEM supplemented with $10 \%$ FBS. All normal cultures were maintained at $37^{\circ} \mathrm{C}$ in saturated humidified incubator with $5 \% \mathrm{CO}_{2}$.

Stable cell transfection with IL-6 short hairpin RNA. Four IL-6targeted short hairpin RNAs (IL-6-shRNAs), as well as one negative control mismatch sequence (IL-6-NC-shRNA), were synthesized by Sangon Biotech Co., Ltd. (Shanghai, China). The targeting shRNA sequences containing recognition sites for $B a m \mathrm{HI}$ in the forward primer and EcoRI in the reverse primers are shown in Table I (Primers 1-5). Subsequently, the pGMLVSC7-IL-6-shRNA lentiviral vectors were constructed using double restriction enzyme digestion and gene recombination. The sequence of these lentivectors was confirmed using direct sequencing. Subsequently, 293T cells were transiently transfected with the recombinant lentivectors, Lenti-HG Mix and HG transgene reagent as described in the manufacturer's manual. The packaging virus was collected from the cell supernatant through lysis after 3 consecutive freeze-thaw cycles. The viral titer was approximately $1 \times 10^{9} \mathrm{TU} / \mathrm{ml}$, which was tested using end- point dilution assay. The LO2 cells were then steadily transfected with lentivirus particles at a multiplicity of infection (MOI) of 20, and the green fluorescence protein in the cells was observed using a laser fluorescence microscope (serial no. N-STORM; Nikon, Tokyo, Japan). Following transfection, the cells were subjected to drug screening with the corresponding antibiotics.

Subsequently, $I L-6$ expression was measured by RT-qPCR as described in the section entitled 'RNA isolation and quantitative RT-PCR'. The primer sequences of the target gene $(I L-6)$ and housekeeping gene $(G A P D H)$ are listed in Table I (Primers 6 and 8). Compared with the control group, the mRNA expression of $I L-6$ was reduced by $73,80,78$ and $74 \%$ in the cells transfected with $I L-6$-shRNA 1, 2, 3 and 4, respectively, when measured at $48 \mathrm{~h}$ post-transfection (Fig. 3A). Therefore, the lentiviral particles harboring $I L-6$-shRNA2 were selected for use in the subsequent experiments.

Stable cell transfection and clone selection. First, total DNA was extracted from the LO2 cells using an AllPrep DNA/RNA mini kit according to the manufacturer's instructions. A 751-bp sequence $(-635$ to +116$)$ of the $I L-6$ promoter region was amplified from the genomic DNA of LO2 homozygous for the $-572 \mathrm{G}$ allele at the rs1800796 locus (18). Specific primer sequences for the 751-bp amplification contained recognition sites for $\mathrm{ClaI}$ in the forward primer and EcoRI in the reverse primer. The 751-bp fragments containing the $-572 \mathrm{G}$ allele represented the wild-type promoters derived from the LO2 cells. The fragments carrying the $-572 \mathrm{G}$ allele and the pGMLV-PA2 lentiviral vectors were doubly digested with restriction endonuclease EcoRI and ClaI. The cytomegalovirus promoter was removed during this process in the linear lentiviral vector. Subsequently, the linear lentivectors and 751-bp fragments were ligated with T4 DNA ligase. The resulting lentivectors were named p-ProG, which carried the $-572 \mathrm{G}$ allele. The $\mathrm{G}$ allele at the $I L-6$ rs 1800796 locus was sitespecifically mutated to $\mathrm{C}$ with the template of wild-type promoter and the QuickChange ${ }^{\mathrm{TM}}$ Site-Directed Mutagenesis kit, as previously described (18). The p-ProC plasmids were synthesized as the p-ProG ones. Specific primer sequences and the plasmid profile for constructing p-ProG and p-ProC are listed in Table I (Primers 9 and 10) and Fig. 1A and B. The sequence of p-ProG and $\mathrm{p}$-ProC was confirmed using direct DNA sequencing.

Second, nonsense mutations of $I L$ - 6 -overexpressing fragments were obtained based on the $I L-6$-shRNA2 sequence and the template of p-IL-6(WT) using the QuickChange ${ }^{\mathrm{TM}}$ Site-Directed Mutagenesis kit, as previously described (18). The resulting fragments were named p-NM-IL-6(WT), and guaranteed that $I L-6$ overexpression was not reduced by $I L-6$ shRNA2 in the LO2 cells. In particular, NM of p-NM-IL-6(WT) refers to nonsense mutations. Specific primers used in this process contained recognition sites for ClaI in the forward primer and $E c o$ RI in the reverse primer (Table I; Primers 11 and 12). Later, the p-ProG lentivectors and p-NM-IL-6(WT) were doubly digested with restriction endonucleases EcoRI and BamHI. Subsequently, the linear p-ProG vectors and IL-6-overexpressing fragments were ligated with T4 DNA ligase. The resulting lentiviral vector was named p-ProGIL-6(MT) (Fig. 1C). Subsequently, the p-ProC-IL-6(MT) lentiviral vector (Fig. 1D) was constructed as was the p-ProGIL-6(MT). The altered sequences were verified using direct sequencing before stable cell transfection. 
Third, the negative control $I L$ - 6 -overexpressing lentivirus particles (no expression of IL-6) and $I L$ - 6 -overexpressing lentivirus particles [carrying ProG-IL-6(MT) or ProC-IL-6(MT)] were packaged as pGMLV-SC7-shRNA-IL-6 described in the previous section. Finally, the LO2 cells with an $80 \%$ suppression of basic $I L-6$ were cultured in a 6 -well culture plate at a density of approximately $2 \times 10^{5}$ cells/well for $24 \mathrm{~h}$, and then stably transfected with $I L$ - 6 -overexpressing lentivirus particles at an efficiency of approximately $70 \%$. These stably transfected cells were named LO2C and LO2G, respectively. Similarly, the LO2 cells stably transfected with $I L-6$-NC-shRNA were again transfected with the negative control $I L$ - 6 -overexpressing lentivirus particles. The resulting cells were designated as NC-LO2 (negative control LO2 cells). Following transfection, the cells were subjected to drug screening with the corresponding antibiotics (ampicillin, puromycin, or blasticidin).

Cell model of $H / R$. To induce $H / R$ after ischemia reperfusion in the liver during liver transplantation and resection, the cell model of $\mathrm{H} / \mathrm{R}$ was constructed as follows: cellular hypoxia was maintained by flushing the incubator set with a continuous gas flow comprising a mixture of $95 \% \mathrm{~N}_{2}$ and $5 \% \mathrm{CO}_{2}$, as previously described $(19,20)$. Re-oxygenation was ensured by continuously flushing with humidified a $95 \%$ air and $5 \% \mathrm{CO}_{2}$ gas mixture. This method resulted in an oxygen content of $0.1 \%$ in the chamber. Subsequently, during the logarithmic growth phase of the $\mathrm{LO} 2 \mathrm{C}$ cells, LO2G cells, normal LO2 cells (control LO2) and NC-LO2 cells were inoculated into 96 -well plates or $3.5 \mathrm{~cm}^{2}$ culture dishes, and incubated with normal medium (DMEM supplemented with $10 \%$ FBS) under normoxic conditions (at $37^{\circ} \mathrm{C}$ in a saturated humidified incubator with $5 \% \mathrm{CO}_{2}$ and $95 \%$ air). Each of the cell types was prepared in 4 groups. Following overnight culture, the medium of these 4 cell types was changed to serumfree Krebs-Henseleit-4-(2-hydroxyethyl)-1-piperazineethanesul fonic acid buffer, as previously described (19), and subjected to hypoxia for $6 \mathrm{~h}$. Subsequently, all the cells were transferred to fresh, warm, oxygenated medium and returned to reoxygenation conditions for a further $1,6,12$ and $24 \mathrm{~h}$, represented as H/R1h, H/R6h, H/R12h and H/R24h, respectively. Additionally, another 4 normoxic groups of cells, as the controls, were incubated for $12 \mathrm{~h}$ along with these H/R-exposed cells. Each group mentioned above was treated in triplicate.

\section{Other methods}

Genomic DNA isolation and genotype determination. Genomic DNA was extracted from the 84 liver specimens with an AllPrep DNA/RNA Mini kit according to the manufacturer's instructions. These samples were previously stored at $-80^{\circ} \mathrm{C}$ until genotyping. Genotyping of the SNPs was performed using a Sequenom MassARRAY SNP genotyping platform, as previously described (21). The protocols mainly included DNA and primer preparation, PCR amplification, primer extension, spotting of primer extension products on the SpectroCHIP, and detection of the primer extension products by mass spectrometry.

Construction of tissue microarray and immunohistochemistry assay. Tissue microarray (TMA) was constructed from disease-free liver tissues $(n=84)$ in collaboration with Shanghai Biochip (Shanghai, China) as previously described (22). Immunohistochemistry assay was then performed using the primary anti- $I L-6$ polyclonal antibody (1:600). The secondary antibody was goat anti-rabbit immunoglobulin G. As the negative controls for all the experiments, the primary antibody was omitted.

Immunohistochemical scoring was performed independently in a blinded manner by two pathologists. The scoring standard has been previously described (23).

$R N A$ isolation and quantitative RT-PCR (RT-qPCR). Total RNA from the 84 disease-free liver tissues and all types of LO2 cells was extracted using TRIzol reagent and the mini RNeasy ${ }^{\circledR}$ kit. First, single-stranded cDNAs were synthesized using a SuperScript VILO cDNA Synthesis kit. Subsequently, RT-qPCR was performed using $2 X$ SYBR-Green qPCR mix and a Mastercycler ${ }^{\circledR}$ Ep Realplex. The fold change of relative mRNA copies was expressed as the relative quantification calculated using the $2^{-\Delta \Delta C t}$ method for each group and calculated as previously described (23). Namely, the data were normalized to a housekeeping gene ( $\beta$-actin or GAPDH). The primer sequences of the target gene and housekeeping gene are shown in Table I (Primers 6 to 8, and 13 and 14).

Cell counting kit-8 assay. A total of $2 \times 10^{3} \mathrm{LO} 2 \mathrm{C}$ cells, $\mathrm{LO} 2 \mathrm{G}$ cells, normal LO2 cells (control LO2 cells) and NC-LO2 cells in the logarithmic growth phase were seeded into 96-well plates, and each group was allocated at least 3 wells. Subsequently, the cells were incubated with normal culture medium and subjected to $\mathrm{H} / \mathrm{R}$, and the effects of different $\mathrm{H} / \mathrm{R}$ conditions on the cell growth or cell viability were evaluated using the CCK- 8 assay according to the instructions of the manufacturer. The cells were incubated with CCK-8 (10 $\mu \mathrm{l} / \mathrm{well})$ at $37^{\circ} \mathrm{C}$ for $3 \mathrm{~h}$. The absorbance (value $\mathrm{A}$ ) of individual wells was determined at a $450 \mathrm{~nm}$ wavelength. The formula for calculating cell viability was (value A of treatment group - value A of blank group)/ (value A of control group - value A of blank group) x100.

ELISA for determining the AST level. After being subjected to different $\mathrm{H} / \mathrm{R}$ conditions or normal culture, the degree of $\mathrm{H} / \mathrm{R}$ injury in the LO2 cells was directly quantified by measuring the concentrations of AST in the cell supernatants using ELISA kits according to the manufacturer's instructions. Inter- and intra-detection coefficients of variation were $<10 \%$ and $<5 \%$, respectively.

Western blot analysis. The expression of STAT3 was determined by western blot analysis as previously described (4). In brief, total cellular protein was extracted and separated by electrophoresis on $12 \%$ sodium dodecyl sulfate polyacrylamide gels and transferred onto polyvinylidene difluoride membranes. The membranes were then washed, blocked and incubated with the primary antibodies (1:1,000 dilution for STAT3; 1:1,000 dilution for $\beta$-actin) overnight at $4^{\circ} \mathrm{C}$, and then incubated with an appropriate horseradish peroxidase-conjugated secondary antibodies $(1: 5,000)$. Signals were detected by chemiluminescence using ECL luminous fluid and scanned by Quantity One software. The protein expression of STAT3 was determined by normalization of the gray value of the STAT3 gene to that of the control housekeeping gene ( $\beta$-actin). Then, the resting relative level of STAT3 protein was calculated using the blank control group as a reference sample.

Statistical analysis. All data were analyzed using SPSS version 19.0 statistical software (SPSS Inc., Chicago, IL, USA) and GraphPad Prism version 6.02 (GraphPad Software, San Diego, CA, USA). Continuous variables were reported as 
Table I. Primer sequences used in this study.

\begin{tabular}{|c|c|c|}
\hline No. & Name & Primer sequences \\
\hline Primer 1 & IL-6-shRNA1 & $\begin{array}{l}\text { F: 5'-gatccGCCCTGAGAAAGGAGACATGTTTCAAGAGAACATGTCTCCTTTCTCAGGGCTTTTTTg-3' } \\
\text { R:5'-aattcAAAAAAGCCCTGAGAAAGGAGACATGTTCTCTTGAAACATGTCTCCTTTCTCAGGGCg-3' }\end{array}$ \\
\hline Primer 2 & IL-6-shRNA2 & $\begin{array}{l}\text { F: 5'-gatcc } G G A T G C T T C C A A T C T G G A T T C T T C A A G A G A G A A T C C A G A T T G G A A G C A T C C T T T T T T g-3 ' \\
\text { R:5'-aattcAAAAAAGGATGCTTCCAATCTGGATTCTCTCTTGAAGAATCCAGATTGGAAGCATCCg-3' }\end{array}$ \\
\hline Primer 3 & IL-6-shRNA3 & $\begin{array}{l}\text { F: 5'-gatccGGAGTTTGAGGTATACCTAGATTCAAGAGATCTAGGTATACCTCAAACTCCTTTTTTg-3' } \\
\text { R:5'-aattcAAAAAAGGAGTTTGAGGTATACCTAGATCTCTTGAATCTAGGTATACCTCAAACTCCg-3' }\end{array}$ \\
\hline Primer 4 & IL-6-shRNA4 & $\begin{array}{l}\text { F: 5'-gatccGCAGGACATGACAACTCATCTTTCAAGAGAAGATGAGTTGTCATGTCCTGCTTTTTTg-3' } \\
\text { R:5'-aattcAAAAAAGCAGGACATGACAACTCATCTTCTCTTGAAAGATGAGTTGTCATGTCCTGCg-3' }\end{array}$ \\
\hline Primer 5 & $\begin{array}{l}\text { IL-6-NC- } \\
\text { shRNA }\end{array}$ & $\begin{array}{l}\text { F: 5'-gatccGTTCTCCGAACGTGTCACGTTTCAAGAGAACGTGACACGTTCGGAGAACTTTTTTACGCGTg-3' } \\
\text { R:5'-aattcACGCGTAAAAAAGTTCTCCGAACGTGTCACGTTCTCTTGAAACGTGACACGTTCGGAGAACg-3' }\end{array}$ \\
\hline Primer 6 & Cell IL-6 & $\begin{array}{l}\text { F: 5'-CAGACAGCCACTCACCTCTTCAG-3' } \\
\text { R: 5'-CTGCCAGTGCCTCTTTGCTG-3' }\end{array}$ \\
\hline Primer 7 & Cell STAT3 & $\begin{array}{l}\text { F: 5'-CTTTGAGACCGAGGTGTATCACC-3' } \\
\text { R:5'-GGTCAGCATGTTGTACCACAGG-3' }\end{array}$ \\
\hline Primer 8 & Cell GAPDH & $\begin{array}{l}\text { F: 5'-GTCTCCTCTGACTTCAACAGCG-3' } \\
\text { R:5'-ACCACCCTGTTGCTGTAGCCAA-3' }\end{array}$ \\
\hline Primer 9 & p-ProG & $\begin{array}{l}\text { F: 5'-GGCC atcgatTCTAAGTGGGCTGAAGCAGGTGAAG-3' } \\
\text { R: 5'-GCCGgaattcTGGGCTCCTGGAGGGGA-3' }\end{array}$ \\
\hline Primer 10 & p-ProC & 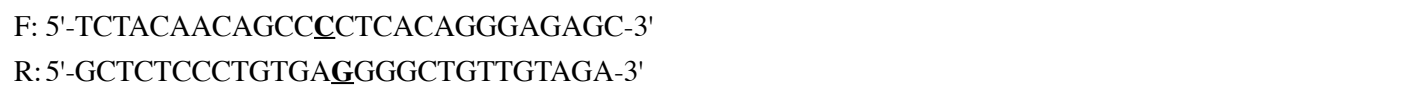 \\
\hline Primer 11 & IL-6(WT) & $\begin{array}{l}\text { F: 5'-CCGgaatccGCCACC ATG AACTCCTTCTCCACAAGCGC-3' } \\
\text { R:5'-CCGggatccCTACATTTGCCGAAGAGCCCTCA-3' }\end{array}$ \\
\hline Primer 12 & IL-6(MT) & 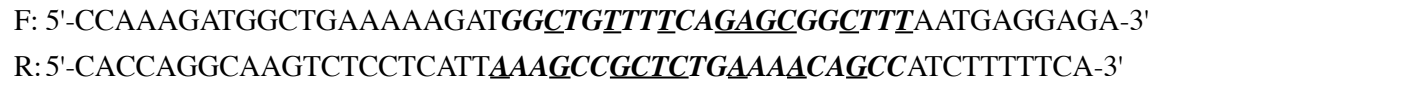 \\
\hline Primer 13 & $\begin{array}{l}\text { IL-6 in } \\
\text { liver tissue }\end{array}$ & $\begin{array}{l}\text { F: 5'-GACCCAACCACAAATGCCAG-3' } \\
\text { R: 5'-ACATTTGCCGAAGAGCCCTC-3' }\end{array}$ \\
\hline Primer 14 & $\begin{array}{l}\beta \text {-actin in } \\
\text { liver tissue }\end{array}$ & $\begin{array}{l}\text { F: 5'-CTCCATCCTGGCGCTGT-3' } \\
\text { R: 5'-GCTGTCACCTTCACCGTTCC-3' }\end{array}$ \\
\hline
\end{tabular}

BamHI, ClaI, EcoRI and nonsense mutations sites and polymorphism position were introduced into the sense and antisense primer as indicated by the underlined text. IL-6, interleukin-6; STAT3, signal transducer and activator of transcription 3; GAPDH, glyceraldehyde-3-phosphate dehydrogenase; F, forward; R, reverse. Bold text indicates base sequences. Italic letters highlight the bases of nonsense mutations different from IL-6-shRNA2. Restriction enzymes identify the lower case letters.

Table II. IL-6 expression in human disease-free liver tissues in the TMA assay.

\begin{tabular}{lcccc}
\hline & & \multicolumn{2}{c}{ IL-6 expression } & \\
\cline { 3 - 4 } Groups & $\begin{array}{c}\text { No. of } \\
\text { case }\end{array}$ & $\begin{array}{c}\text { Weak } \\
(\%)\end{array}$ & $\begin{array}{c}\text { Intermediate } \\
(\%)\end{array}$ & P-value \\
\hline CC & 39 & $11(28.2)$ & $28(71.8)$ & 0.006 \\
CG/GG & 45 & $26(57.8)$ & $19(42.2)$ & \\
\hline
\end{tabular}

IL-6, interleukin-6; TMA, tissue microarray.

the means \pm standard deviation (SD) and examined using the Student's t-test or ANOVA. Categorical data were analyzed using the Chi-square test. Two-sided probability values were used for all statistical tests. Significance was established at the $\mathrm{P}<0.05$ level.

\section{Results}

Distribution of genotypes and alleles. The frequencies of genotypes CC, CG and GG were 46.4, 47.6 and 6.0\%, respectively. The allele $\mathrm{C}$ and $\mathrm{G}$ frequencies were 70.2 and $29.8 \%$ (data not shown). The genotype CG/GG carriers were pooled into one group, as previously described (16) (Table II). There were no significant differences between the CC group and CG/ GG group with regard to race, gender, age distribution and body mass index $(\mathrm{P}>0.05$; data not shown).

Expression of IL-6 in human disease-free liver tissues. The expression of $I L-6$ in the 84 human disease-free liver tissues was evaluated using the TMA. The panels in Fig. 2A1-A3 and B1-B3 

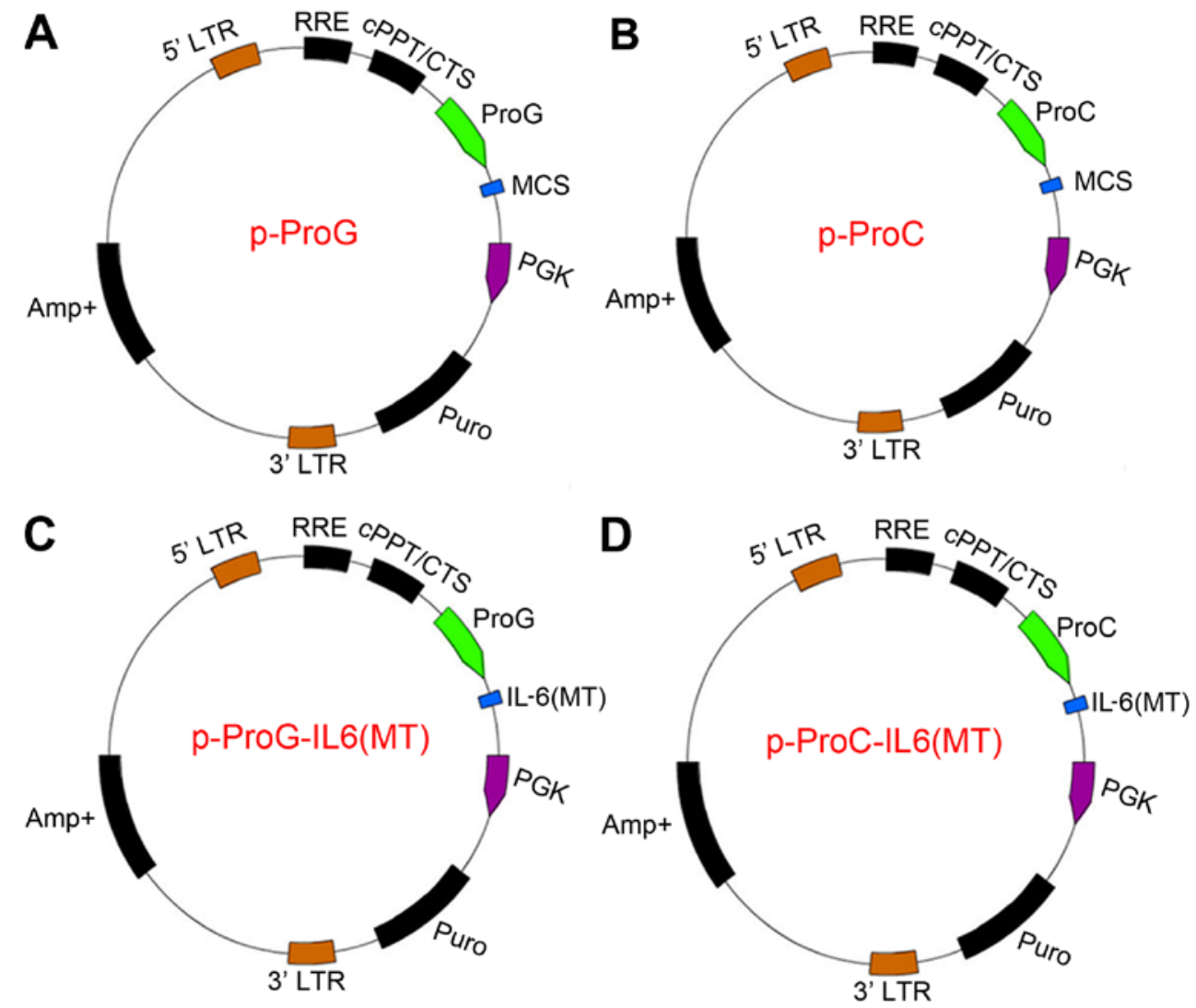

Figure 1. Lentiviral vector profile constructed and used in the present study. (A) Lentivector profile of completed construction containing $-572 \mathrm{G}$ at rs1800796 locus. (B) Lentivector profile of completed construction containing -572C. (C) Lentivector profile of completed construction containing $-572 \mathrm{G}$ and $I L-6$ overexpression fragment. (D) Lentivector profile of completed construction containing $-572 \mathrm{C}$ and $I L-6$ overexpression fragment. IL-6, interleukin-6.

represent the intermediate and weak immunostaining profiles of IL-6 protein in hepatocytes. Among the 45 genotype (CG/GG) carriers, 57.8\% (26/45) of the specimens exhibited weak staining and $42.2 \%(19 / 45)$ were intermediately immunoreactive for IL-6 (Table II). However, of the 39 genotype CC carriers, there was differential $I L-6$ expression, with intermediate staining in $71.8 \%$ (28/39) of the samples and weak staining in $28.2 \%(11 / 39)$ of the samples $(\mathrm{P}=0.006)$ (Table II). Subsequently, RT-qPCR analysis was undertaken to further determine the $I L-6 \mathrm{mRNA}$ levels in the 84 liver tissue samples. The $\Delta \mathrm{Ct}$ value for the genotype $\mathrm{CC}$ carriers was less than that for the $\mathrm{CG} / \mathrm{GG}$ carriers (6.7 \pm 1.2 vs. $7.6 \pm 1.3, \mathrm{P}=0.002$ ) (Fig. $2 \mathrm{C}$ ), confirming the results of immunostaining analysis. Further, when comparing the results of RT-qPCR between all the intermediate samples and all weak staining samples, the difference was more obvious $(\mathrm{P}<0.001)$. The protein (staining intensity) and $I L-6$ mRNA expression were well correlated.

Construction of $\mathrm{LO} 2$ cell lines. The $\mathrm{LO} 2 \mathrm{C}$ cells and $\mathrm{LO} 2 \mathrm{G}$ cells, stably transfected with IL-6-shRNA2 lentivirus particles and $I L-6$ overexpression lentivirus particles, were successfully constructed. Therefore, the $\mathrm{LO} 2 \mathrm{C}$ and $\mathrm{LO} 2 \mathrm{G}$ cells produced ectopic $I L-6$ overexpression and approximately $20 \%$ of basic IL-6. Similarly, the NC-LO2 cells were constructed by stable transfection with IL-6-NC-shRNA and negative control $I L$-6-overexpressing lentiviral particles. The foregoing stable transfection protocol was described in the Materials and methods. Notably, the NC-LO2 cells served as the negative controls. Normal LO2 cells were used as blank controls. The LO2G cells contained the wild-type promoter derived from the LO2 cells, and the WT promoter for the control compared with the $\mathrm{LO} 2 \mathrm{C}$ cells. The double transfection of lentiviral particles did not alter the cell morphology of the LO2 cells significantly compared with the normal LO2 cells (Fig. 3B and C) at $48 \mathrm{~h}$. Additionally, fluorescence analysis of the $\mathrm{LO} 2$ cells revealed an infection lentiviral transduction efficiency of approximately $70 \%$ in accordance with the manufacturer's instructions. There were no cells in the blank zone, and the fluorescence was $100 \%$ after $0.5 \mu \mathrm{g} / \mathrm{ml}$ puromycin selection (Fig. 3D).

$H / R$ injury level of LO2 cells. Compared with the normoxic group cells, the concentrations of AST (ng/ml) in the LO2 cells increased significantly in all the H/R-exposed groups ranging from 30.18 to $78.98 \%$, suggesting varying degrees of damage in the LO2 cells (Fig. 4A). In addition, the concentrations of AST in the $\mathrm{LO} 2 \mathrm{G}$ cells were much higher than those in the $\mathrm{LO} 2 \mathrm{C}$ cells at $\mathrm{H} / \mathrm{R} 6 \mathrm{~h}, \mathrm{H} / \mathrm{R} 12 \mathrm{~h}$ and $\mathrm{H} / \mathrm{R} 24 \mathrm{~h}(\mathrm{P}=0.003, \mathrm{P}=0.041$ and $\mathrm{P}<0.001$, respectively) (Fig. 4A). The differences between the experimental groups ( $\mathrm{LO} 2 \mathrm{C}$ and $\mathrm{LO} 2 \mathrm{G}$ ) and the control groups (control LO2 and NC-LO2) were more significant at all $\mathrm{H} / \mathrm{R}$ exposure time points $(\mathrm{P}<0.01)$. However, there was no obvious difference between the control LO2 and NC-LO2 cells, which suggested that the lentiviral particles had a decreased effect on $\mathrm{H} / \mathrm{R}$ injury in LO2 cells. This difference between the LO2C and $\mathrm{LO} 2 \mathrm{G}$ cells was mainly caused by the sequence variants of the IL-6 rs1800796 locus SNPs $(\mathrm{G} \rightarrow \mathrm{C})$. 

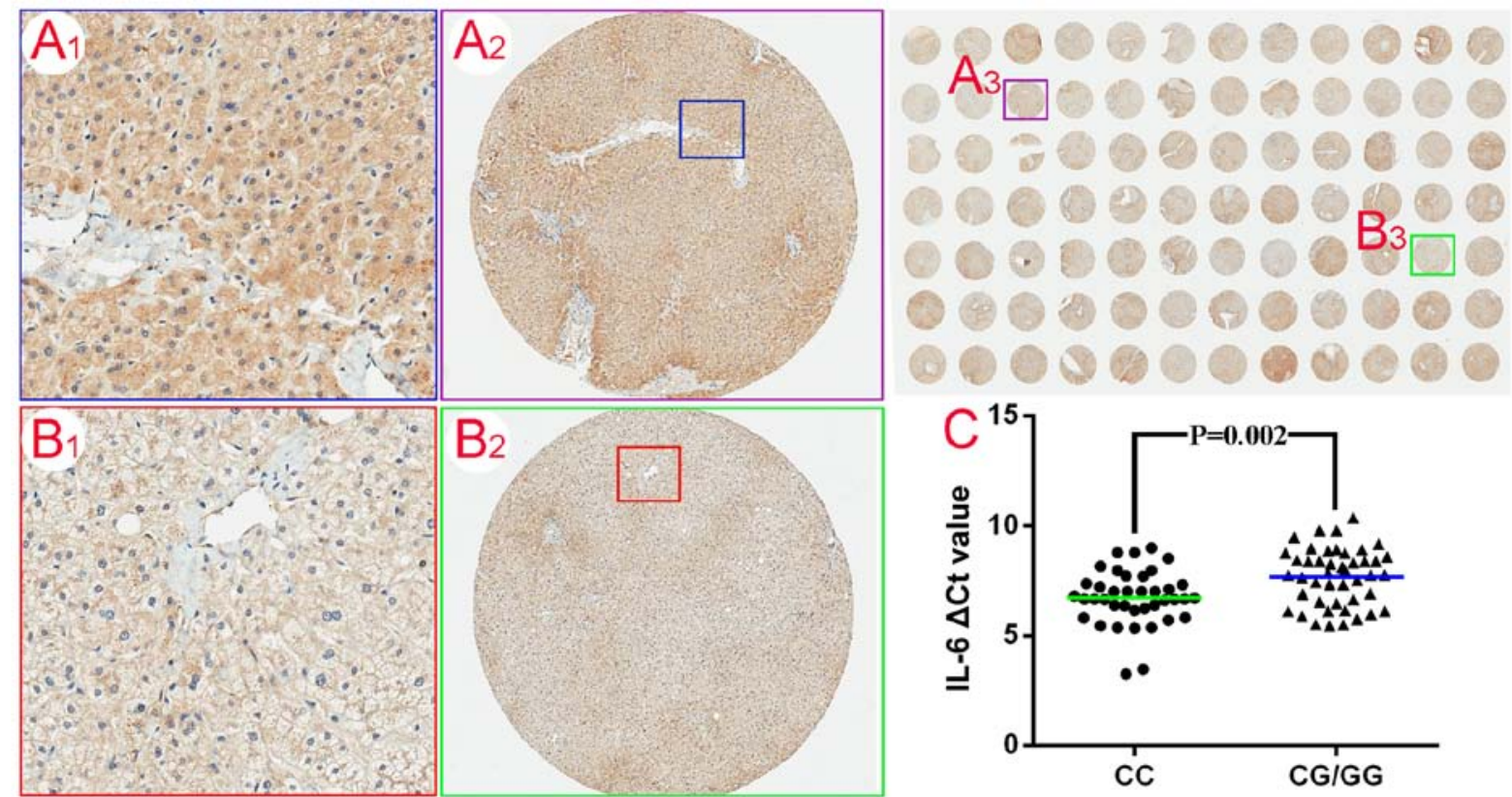

Figure 2. IL-6 expression in normal human liver tissues (n=84). (A1-A3) Intermediate staining of IL-6 in normal liver tissues (original amplification, x200, x40, $\mathrm{x} 4$, respectively). (B1-B3) Weak staining of IL-6 in normal liver tissues (original amplification, x200, x40, x4, respectively). (C) $I L-6$ mRNA expression was determined using RT-qPCR according to genotypes of the 84 disease-free tissues. IL-6, interleukin-6.
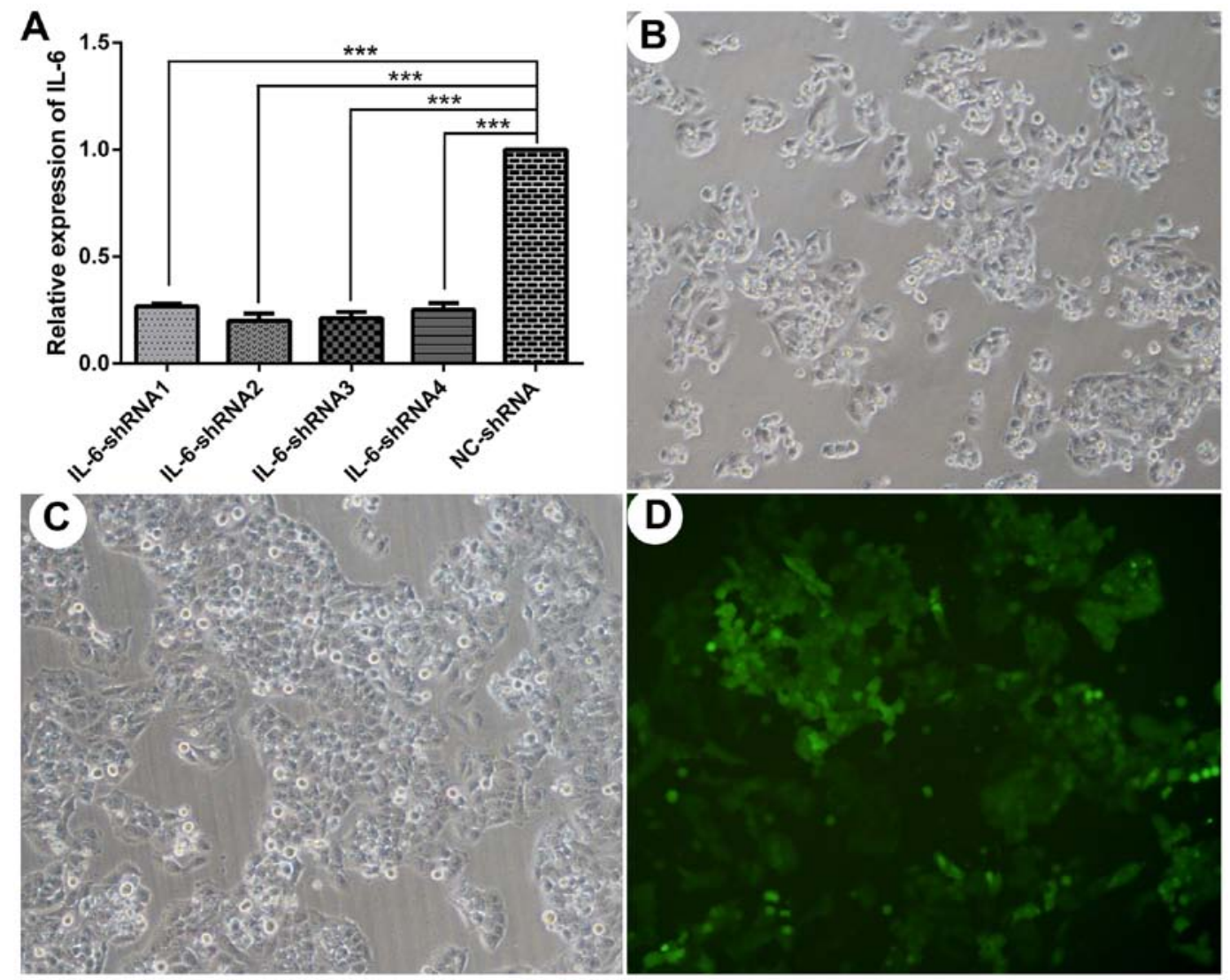

Figure 3. Relative expression of $I L-6$ and images of LO2 cells (original amplification, x10). (A) Relative expression of $I L-6$ examined by RT-qPCR after transfection of the LO2 cells with lentiviral particles of control (IL-6-NC-shRNA) and $I L$ - 6 -shRNAs. (B) Bright-field images of LO2 cells before lentiviral transduction. (C) Bright-field images of LO2 cells at $48 \mathrm{~h}$ after lentiviral transduction. (D) Fluorescent image of LO2 cells at $48 \mathrm{~h}$ after lentiviral transduction. All data are expressed as the means $\pm \mathrm{SD}$ ( $\mathrm{n}=3$ experiments). ${ }^{* * *} \mathrm{P}<0.001$ compared with control group. IL-6, interleukin-6; NC-shRNAs, negative control-short hairpin RNAs.

Viability of LO2 cells. Following H/R injury, the rate of survival in the 4 groups of cells was significantly decreased by approximately $45 \%$ compared with the normoxic group of cells. In addition, the viability of the $\mathrm{LO} 2 \mathrm{C}$ cells was signfi- 

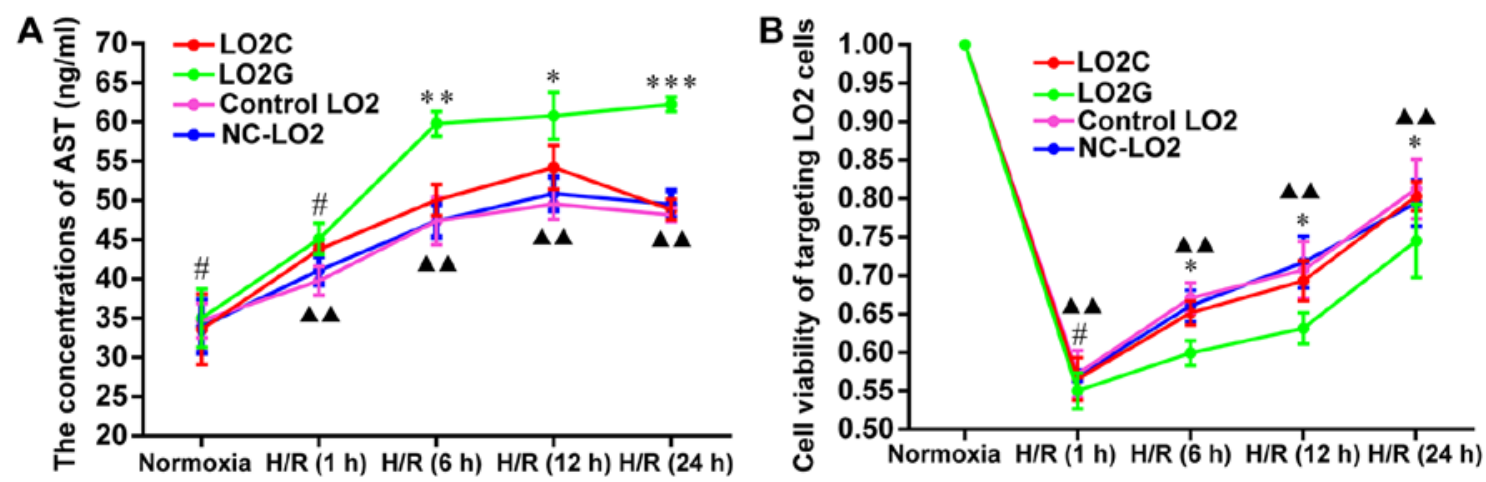

Figure 4. Role of $I L-6$ rs1800796 SNPs in LO2 cells. (A) Concentrations of AST in the supernatant of LO2 cells in normoxic and H/R groups. (B) Cell viability of LO2 cells in in normoxic and H/R groups. All data are expressed as the means $\pm \mathrm{SD}$ ( $\mathrm{n}=3$ experiments). ${ }^{* * * *} \mathrm{P}<0.001,{ }^{* *} \mathrm{P}<0.01,{ }^{*} \mathrm{P}<0.05$ and ${ }^{~} \mathrm{P}>0.05$ between LO2G and LO2C groups and ${ }^{\wedge} \mathrm{P}<0.01$ between the experimental group and the control groups. AST, alanine aminotransferase; H/R, hypoxia/reoxygenation; IL-6, interleukin-6; SNP, single nucleotide polymorphism.
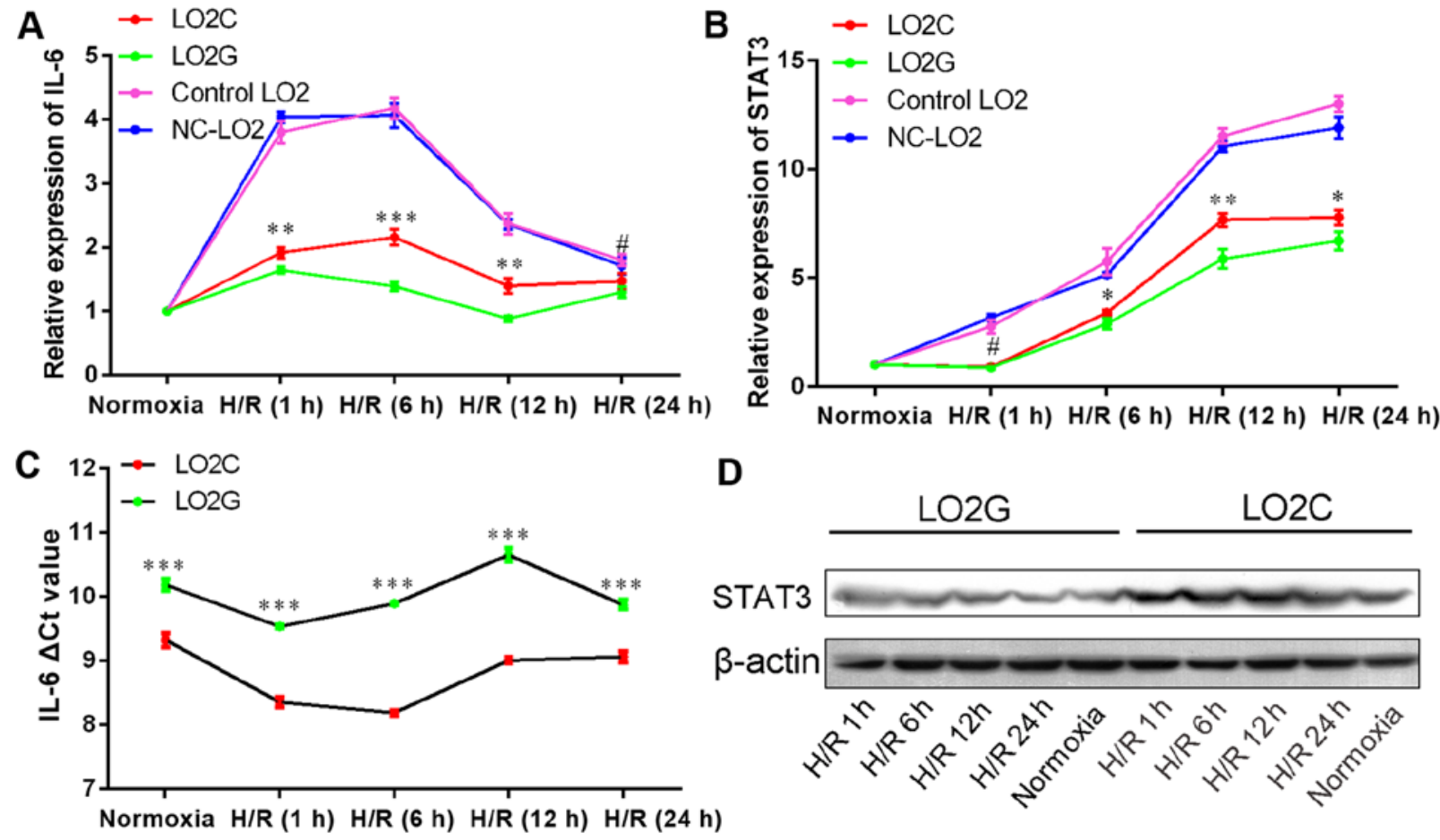

D

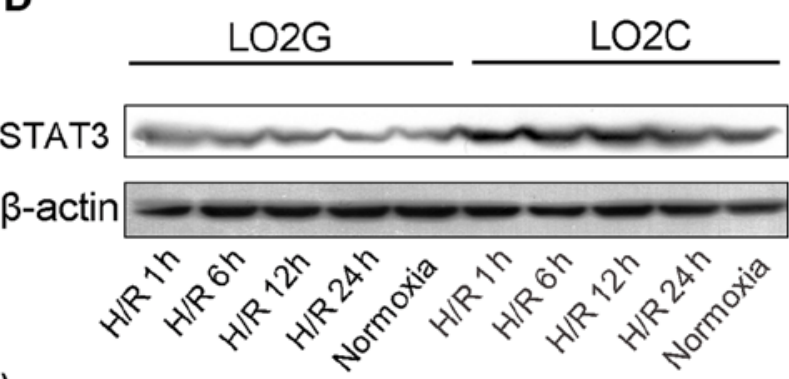

Figure 5. Effects of $I L-6$ rs1800796 SNPs on the expression of $I L-6$ and STAT3 in normoxic and H/R groups. (A) Relative expression of $I L-6$ detected using RT-qPCR. (B) Relative expression of STAT3 detected using RT-qPCR. (C) $\triangle \mathrm{Ct}$ values of $I L-6$. (D) Levels of expression of STAT3 protein were determined by western blot analysis. All data are expressed as the means $\pm \mathrm{SD}(\mathrm{n}=3) .{ }^{* * * *} \mathrm{P}<0.001,{ }^{* * *} \mathrm{P}<0.01,{ }^{*} \mathrm{P}<0.05$ and ${ }^{*} \mathrm{P}>0.05$ comparing LO2G and LO2C groups. H/R, hypoxia/ reoxygenation; IL-6, interleukin-6; STAT3, signal transducer and activator of transcription; SD, standard deviation; SNP, single nucleotide polymorphism.

cantly increased compared to that of the LO2G cells from $\mathrm{H} /$ R6h to $\mathrm{H} / \mathrm{R} 24 \mathrm{~h}(\mathrm{P}=0.034, \mathrm{P}=0.016$ and $\mathrm{P}=0.031)$ (Fig. 4B). In addition, there were significant differences between the experimental group and the control group at all H/R exposure time points $(\mathrm{P}<0.01)$ (Fig. 4B). There were no obvious differences in cell viability between the control LO2 and NC-LO2 cells, which suggested that the lentiviral particles had less prominent effects on the biological activity of the LO2 cells. The results were consistent with the those obtained for the AST levels.

Expression of IL- 6 and STAT3 in LO2 cells. Compared with $I L-6$ expression in the normoxic groups, the increase in the mRNA expression of $I L-6$ in the $\mathrm{LO} 2 \mathrm{C}$ cells was greater than that in the LO2G cells from $\mathrm{H} / \mathrm{R} 1 \mathrm{~h}$ to $\mathrm{H} / \mathrm{R} 24 \mathrm{~h}(\mathrm{P}=0.009, \mathrm{P}<0.001$, $\mathrm{P}=0.002$ and $\mathrm{P}<0.05$, respectively) (Fig. 5A). Similarly, STAT3 expression also increased from $\mathrm{H} / \mathrm{R} 1 \mathrm{~h}$ to $\mathrm{H} / \mathrm{R} 24 \mathrm{~h}$ ( $\mathrm{P}<0.05$, $\mathrm{P}=0.034, \mathrm{P}=0.004$ and $\mathrm{P}=0.027$ ) (Fig. $5 \mathrm{~B})$. Additionally, relative to the housekeeping gene $(G A P D H), I L-6$ expression was lower in the $\mathrm{LO} 2 \mathrm{G}$ cells than in the $\mathrm{LO} 2 \mathrm{C}$ cells under normoxic conditions $(\mathrm{P}<0.001)$ (Fig. 5C). This result was consistent with that of $I L-6$ expression in human disease-free liver tissues. We also investigated whether STAT3 protein expression was increased under H/R conditions by conducting subsequent western blot analysis, which confirmed that the STAT3 level was also higher at all time points. Additionally, STAT3 expression was higher in the LO2C cells compared with the LO2G cells (Fig. 5D). 
The result was confirmed by densitometry (integral optical density, IOD) to measure the STAT3 levels from H/R1 to H/R24 $(\mathrm{P}<0.05)$.

\section{Discussion}

Hepatic IRI caused by H/R is a common pathological phenomenon during liver surgery, liver transplantation and severe liver trauma (1-4). The simultaneous activation and interaction of multiple cytokines, particularly multifunctional IL-6, regulate cell injury and death during liver regeneration. $I L-6$ plays an essential role in triggering liver regeneration during the priming phase (24). Furthermore, protective $I L-6 / S T A T 3$ signaling is initiated within minutes to hours after partial liver hepatectomy (4,25). Recently, the $I L-6$ rs1800796 locus SNPs were thoroughly investigated in a healthy population (13), and under several clinical conditions (26-28). It has been reported that only donor $I L-6$ rs1800796 SNPs affect the metabolism of tacrolimus, mainly by influencing liver function and regeneration following liver transplantation (16). Therefore, in this study, we examined IL-6 expression in 84 disease-free liver tissues using TMA. The IL-6 level was higher in genotype $\mathrm{CC}$ carriers than in genotype $\mathrm{CG} / \mathrm{GG}$ carriers, due to a higher intermediate expression rate $(\mathrm{P}=0.006)$ (Fig. $2 \mathrm{~A}$ and $\mathrm{B}$ and Table II). Subsequently, it was verified at the mRNA level (Fig. 2C). Therefore, allele C promotes $I L-6$ transcription efficiency, which is consistent with a study involving basic promoter experiments using a reporter gene system (18). As shown in Fig. 2A and B, the IL-6 was mainly expressed in the cytoplasm of hepatocytes. It has been reported that approximately $70 \%$ of liver cells are hepatocytes (29). Furthermore, studies have suggested that primary rodent and LO2 hepatocytes synthesize and secrete IL-6 $(9,30,31)$. Therefore, the biological mechanisms of $I L-6$ and its rs1800796 locus SNPs, in human hepatocytes, warrant further investigation.

The immortalized human normal liver cell line, LO2, was derived from primary normal human hepatocytes, and still retains the biological characteristics of normal adult hepatocytes (17). Additionally, the current third-generation lentivirus used in this study, proved that human immunodeficiency virus-based vectors are valid manufacturing tools for stable genetic modification $(32,33)$. The $\mathrm{LO} 2 \mathrm{G}$ cells and $\mathrm{LO} 2 \mathrm{C}$ cells successfully generated via lentivirus-mediated method stably produced ectopic IL-6 overexpression and approximately $20 \%$ of endogenic IL- 6 . The LO2G and LO2C cells, respectively, carried allele $\mathrm{G}$ or $\mathrm{C}$ at the $I L-6$ rs 1800796 locus. The NC-LO2 cells, as the negative control group, were also successfully constructed. Furthermore, cell morphology was not altered significantly following double lentivirus transfection (Fig. 3B and C). Subsequently, based on the LO2C cells, the LO2G cells, normal LO2 cells (blank control group) and NC-LO2 cells, a cell model of $\mathrm{H} / \mathrm{R}$ was successfully established in order to examine H/R injury $(19,20)$.

In this study, the concentrations of AST were higher in the $\mathrm{LO} 2 \mathrm{C}$ cells than in the LO2G cells from H/R6h to $\mathrm{H} /$ R24h ( $\mathrm{P}<0.05)$ (Fig. 4A). Similarly, there were also marked differences between the $\mathrm{LO} 2 \mathrm{C}$ and $\mathrm{LO} 2 \mathrm{G}$ cells as regards cell viability from $\mathrm{H} / \mathrm{R} 6 \mathrm{~h}$ to $\mathrm{H} / \mathrm{R} 24 \mathrm{~h}(\mathrm{P}<0.05)$ (Fig. 4B). The differences in the AST data or cell viability between the experimental and the control groups were significant at all
$\mathrm{H} / \mathrm{R}$ time-points $(\mathrm{P}<0.01)$ (Fig. 4). Interestingly, these data did not differ between the control LO2 and NC-LO2 cells, which suggested that the lentiviral particles exerted less prominent effects on the biological properties of LO2 cells. Therefore, the differences between LO2C and LO2G cells were mainly attributed to allele $\mathrm{G}$ mutating into $\mathrm{C}$ at the rs1800796 locus.

The relative increase in IL-6 expression in the $\mathrm{LO} 2 \mathrm{C}$ cells was higher than that in the $\mathrm{LO} 2 \mathrm{G}$ cells from $\mathrm{H} / \mathrm{R} 1 \mathrm{~h}$ to $\mathrm{H} /$ R12h (Fig. 5A). LO2C cells expressed greater IL-6 than LO2G cells in the normoxia group $(\mathrm{P}<0.001)(\mathrm{Fig} .5 \mathrm{C})$. The expression of STAT3, downstream signal molecules of IL-6, in LO2C cells increased higher than in LO2G cells, from H/R6h to H/R24h $(\mathrm{P}<0.05)$ (Fig. 5B). Similarly, it was also found that the STAT3 level was higher at all time-points under H/R conditions. This phenomenon was more obvious in LO2C cells compared with LO2G cells (Fig. 5D) and confirmed by grey level of STAT3 from $\mathrm{H} / \mathrm{R} 1$ to $\mathrm{H} / \mathrm{R} 24(\mathrm{P}<0.05)$.

The results suggest that LO2 cells synthesized IL-6, and the subsequent IL-6/STAT3 signaling pathway was triggered within minutes to hours following H/R injury. IL-6 exerted hepatoprotective effects and decreased cell activity through the upregulation of IL-6/STAT3 signaling. Although IL-6 production by non-parenchymal cells, such as Kupffer cells may play a role $(8,9)$, these findings suggested that IL-6 produced in hepatocytes promoted the activation of the IL-6/STAT3 signaling pathway and also exerted hepatoprotective effects in an autocrine manner.

It was observed that $I L-6$ rs1800796 SNPs affected $I L-6$ transcription efficiency under normoxic or H/R conditions. Furthermore, the allele $\mathrm{G} \rightarrow \mathrm{C}$ substitution accelerated the production of IL-6 and attenuated H/R injury in hepatocytes. These SNPs were present in the $I L-6$ promoter region, and altered the affinity with the regulatory protein, and subsequently influenced $I L-6$ transcription (15). For example, SNPs in the osteopontin promoter region have been shown to alter DNA binding affinity to transcription factors SP1/SP3 (34).

The present study has several limitations. First, although the use of TMAs enables the analysis of 84 samples in a relatively short period of time, the results may be unreliable due to the small sample size. Thus, these results need to be confirmed with a larger population. Second, in vitro studies using the LO2 cell line, but not adult hepatocytes, may be confounded by the cell type and culture conditions. Therefore, further in vivo studies are warranted in order to elucidate the function of $I L-6$ gene and SNPs. Third, this study mainly investigated the changes in STAT3 and IL-6 RNA or protein levels. Although many studies have fully established the association between IL- 6 protein and mRNA expression, its functional significance requires further analysis. Nevertheless, our results provide insight into the potential role of $I L-6$ and its SNPs in human hepatocytes.

In conclusion, this study, to the best of our knowledge, was the first of its kind to demonstrate that sequence variants of $I L-6$ rs1800796 locus SNPs $(\mathrm{G} \rightarrow \mathrm{C})$ increase the transcription efficiency of the $I L-6$ gene and protect hepatocytes following H/R. H/R injury triggered the synthesis of IL-6 and STAT3 in the liver parenchymal cells. Our results facilitate efficient liver regeneration and restoration following liver surgery. Additionally, these data establish the framework for exploring the role of the $I L-6$ gene and its polymorphisms in other H/R conditions, such as those affecting the brain and kidneys. 


\section{Acknowledgements}

The authors of this study would like to thank Lijuan Feng (GenomeDitech Co.,Ltd.,Shanghai,China) for her experimental and technical advice. This study was supported by the National Natural Science Foundation of China (grant no. 81370579) and the Leading Project of Science, Technology Commission of Shanghai Municipality (grant no. 134119a6300).

\section{References}

1. Serracino-Inglott F, Habib NA and Mathie RT: Hepatic ischemia-reperfusion injury. Am J Surg 181: 160-166, 2001.

2. Klune JR and Tsung A: Molecular biology of liver ischemia/reperfusion injury: Established mechanisms and recent advancements. Surg Clin North Am 90: 665-677, 2010.

3. Hiranuma S, Ito K, Noda Y, Ozasa H, Koike Y and Horikawa $\mathrm{S}$ : Amelioration of hepatic ischemia/reperfusion injury in the remnant liver after partial hepatectomy in rats. J Gastroenterol Hepatol 22: 2167-2172, 2007.

4. Taki-Eldin A, Zhou L, Xie HY and Zheng SS: Liver regeneration after liver transplantation. Eur Surg Res 48: 139-153, 2012.

5. Streetz KL, Luedde T, Manns MP and Trautwein C: Interleukin 6 and liver regeneration. Gut 47: 309-312, 2000.

6. Tiberio L, Tiberio GA, Bardella L, Cervi E, Cerea K, Dreano M, Garotta G, Fra A, Montani N, Ferrari-Bravo A, et al: Mechanisms of interleukin- 6 protection against ischemia-reperfusion injury in rat liver. Cytokine 34: 131-142, 2006.

7. da Silva CG, Studer P, Skroch M, Mahiou J, Minussi DC, Peterson CR, Wilson SW, Patel VI, Ma A, Csizmadia E and Ferran : A20 promotes liver regeneration by decreasing SOCS3 expression to enhance IL-6/STAT3 proliferative signals. Hepatology 57: 2014-2025, 2013.

8. Selzner N, Selzner M, Odermatt B, Tian Y, Van Rooijen N and Clavien PA: ICAM-1 triggers liver regeneration through leukocyte recruitment and Kupffer cell-dependent release of TNF-alpha/IL-6 in mice. Gastroenterology 124: 692-700, 2003.

9. Yoshiya S, Shirabe K, Imai D, Toshima T, Yamashita Y, Ikegami T, Okano S, Yoshizumi T, Kawanaka H and Maehara Y: Blockade of the apelin-APJ system promotes mouse liver regeneration by activating Kupffer cells after partial hepatectomy. J Gastroenterol 50: 573-582, 2015.

10. Kämäräinen OP, Solovieva S, Vehmas T, Luoma K, Riihimäki H, Ala-Kokko L, Männikkö M and Leino-Arjas P: Common interleukin- 6 promoter variants associate with the more severe forms of distal interphalangeal osteoarthritis. Arthritis Res Ther 10: R21, 2008.

11. Li J, Song J, Jiang MH, Zheng JG, Gao SP, Zhu JH and Pan M: Interleukin-6 promoter polymorphisms and susceptibility to atrial fibrillation in elderly Han Chinese patients with essential hypertension. J Interferon Cytokine Res 32: 542-547, 2012.

12. Gao SP, Liang S, Pan M, Sun RL, Chen C, Luan H and Jiang MH: Interleukin-6 genotypes and serum levels in Chinese Hui population. Int J Clin Exp Med 7: 2851-2857, 2014.

13. Pan M, Gao SP, Jiang MH, Guo J, Zheng JG and Zhu JH: Interleukin 6 promoter polymorphisms in normal Han Chinese population: Frequencies and effects on inflammatory markers. J Investig Med 59: 272-276, 2011.

14. Cheung BM, Ong KL, Tso AW, Leung RY, Cherny SS, Sham PC, Thomas GN, Lam TH and Lam KS; Investigators of the Hong Kong Cardiovascular Risk Factor Prevalence Study: Relationship of plasma interleukin- 6 and its genetic variants with hypertension in Hong Kong Chinese. Am J Hypertens 24: 1331-1337, 2011.

15. Liao PY and Lee KH: From SNPs to functional polymorphism: The insight into biotechnology applications. Biochem Eng J 49: 149-158, 2010.

16. Chen D, Fan J, Guo F, Qin S, Wang Z and Peng Z: Novel single nucleotide polymorphisms in interleukin 6 affect tacrolimus metabolism in liver transplant patients. PLoS One 8: e73405, 2013.
17. Cheng B, Zheng Y, Guo X, Wang Y and Liu C: Hepatitis B viral $\mathrm{X}$ protein alters the biological features and expressions of DNA repair enzymes in LO2 cells. Liver Int 30: 319-326, 2010.

18. Chen J, Liu RY, Yang L, Zhao J, Zhao X, Lu D, Yi N, Han B, Chen XF, Zhang K, et al: A two-SNP IL-6 promoter haplotype is associated with increased lung cancer risk. J Cancer Res Clin Oncol 139: 231-242, 2013.

19. Carini R, De Cesaris MG, Splendore R, Bagnati $M$ and Albano E: Ischemic preconditioning reduces $\mathrm{Na}(+)$ accumulation and cell killing in isolated rat hepatocytes exposed to hypoxia. Hepatology 31: 166-172, 2000.

20. Bhogal RH, Weston CJ, Curbishley SM, Bhatt AN, Adams DH and Afford SC: Variable responses of small and large human hepatocytes to hypoxia and hypoxia/reoxygenation (H-R). FEBS Lett 585: 935-941, 2011.

21. Gabriel S, Ziaugra L and Tabbaa D: SNP genotyping using the Sequenom MassARRAY iPLEX platform. Curr Protoc Hum Genet: Chapter 2: Unit 2.12, 2009.

22. Li D, Yan D, Tang H, Zhou C, Fan J, Li S, Wang X, Xia J, Huang F, Qiu G and Peng Z: IMP3 is a novel prognostic marker that correlates with colon cancer progression and pathogenesis. Ann Surg Oncol 16: 3499-3506, 2009.

23. Yan DW, Li DW, Yang YX, Xia J, Wang XL, Zhou CZ, Fan JW, Wen YG, Sun HC, Wang Q, et al: Ubiquitin D is correlated with colon cancer progression and predicts recurrence for stage II-III disease after curative surgery. Br J Cancer 103: 961-969, 2010.

24. Cressman DE, Greenbaum LE, DeAngelis RA, Ciliberto G, Furth EE, Poli V and Taub R: Liver failure and defective hepatocyte regeneration in interleukin-6-deficient mice. Science 274: 1379-1383, 1996.

25. Jin LM, Jin SF, Liu YX, Zhou L, Xie HY, Yan S, Xu X and Zheng SS: Ischemic preconditioning enhances hepatocyte proliferation in the early phase after ischemia under hemi-hepatectomy in rats. Hepatobiliary Pancreat Dis Int 11: 521-526, 2012.

26. Motoyama S, Nakatsu T, Miura M, Hinai Y, Minamiya Y and Ogawa J: Interleukin-6 -634G $>\mathrm{C}$ genetic polymorphism is associated with prognosis following surgery for advanced thoracic esophageal squamous cell carcinoma. Dig Surg 29: 194-201, 2012.

27. Oishi Y, Watanabe Y, Shinoda S, Naka M, Ozawa Y, Matsuyama T, Morozumi K and Fuke Y: The IL-6 gene polymorphism $-634 \mathrm{C}>\mathrm{G}$ and IL17F gene polymorphism $7488 \mathrm{~T}>\mathrm{C}$ influence bone mineral density in young and elderly Japanese women. Gene 504: 75-83, 2012.

28. Zhang G, Zhou B, Wang W, Zhang M, Zhao Y, Wang Z, Yang L, Zhai J, Feng CG, Wang J and Chen X: A functional singlenucleotide polymorphism in the promoter of the gene encoding interleukin 6 is associated with susceptibility to tuberculosis. J Infect Dis 205: 1697-1704, 2012.

29. Weibel ER, Stäubli W, Gnägi HR and Hess FA: Correlated morphometric and biochemical studies on the liver cell. I. Morphometric model, stereologic methods, and normal morphometric data for rat liver. J Cell Biol 42: 68-91, 1969.

30. Xiang WQ, Feng WF, Ke W, Sun Z, Chen Z and Liu W: Hepatitis B virus $X$ protein stimulates IL- 6 expression in hepatocytes via a MyD88-dependent pathway. J Hepatol 54: 26-33, 2011.

31. Norris CA, He M, Kang LI, Ding MQ, Radder JE, Haynes MM, Yang Y, Paranjpe S, Bowen WC, Orr A, et al: Synthesis of IL-6 by hepatocytes is a normal response to common hepatic stimuli. PLoS One 9: e96053, 2014.

32. Lee CL, Chou M, Dai B, Xiao L and Wang P: Construction of stable producer cells to make high-titer lentiviral vectors for dendritic cell-based vaccination. Biotechnol Bioeng 109: 1551-1560, 2012

33. Li M, Husic N, Lin Y and Snider BJ: Production of lentiviral vectors for transducing cells from the central nervous system. J Vis Exp 24: e4031, 2012.

34. Giacopelli F, Marciano R, Pistorio A, Catarsi P, Canini S, Karsenty G and Ravazzolo R: Polymorphisms in the osteopontin promoter affect its transcriptional activity. Physiol Genomics 20: 87-96, 2004. 